\title{
The neuropsychiatric aspect of the HCV infection
}

\author{
Magdalena Więdłocha, A-D, Piotr Marcinowicz, ${ }^{2, C, D, F}$, Dorota Sokalla ${ }^{3, C, D}$, Bartłomiej Stańczykiewic ${ }^{4, A, ~ E, F}$ \\ ${ }^{1}$ Masovian Specialist Health Center, Pruszków, Poland \\ ${ }^{2}$ Lower Silesian Centre of Lung Diseases, Wrocław, Poland \\ ${ }^{3}$ Department of Pediatric Bone Narrow Transplantation, Oncology and Hematology, Wroclaw Medical University, Poland \\ ${ }^{4}$ Division of Consultation Psychiatry and Neuroscience, Department of Psychiatry, Wroclaw Medical University, Poland \\ A - research concept and design; $\mathrm{B}$ - collection and/or assembly of data; $\mathrm{C}$ - data analysis and interpretation; \\ $D$ - writing the article; $E$ - critical revision of the article; $F$ - final approval of article
}

\section{Address for correspondence \\ Magdalena Więdłocha}

E-mail: mwiedlocha@wp.pl

\section{Funding sources}

none declared

\section{Conflict of interest}

none declared

Received on November 10, 2014

Revised on December 9, 2014

Accepted on June 10, 2015

\begin{abstract}
HCV infection is significantly more prevalent in the population of psychiatric patients, drug addicts and people tending to undertake risky sexual behaviors than in the general population. This article presents a spectrum of psychopathological symptoms and psychological dysfunctions, an outline of current theories on the neuropathology and psychiatric aspects of HCV infection treatment. The unspecific character of the psychopathological symptoms in the HCV infection makes the process of thorough diagnostics and adequate treatment difficult, thus the specific and characteristic features have been emphasized. The aim of this review is to shed light not only on the basic information concerning CNS pathology but also on the conclusions emerging from the studies of different authors, of various methodology, in diverse study groups and also to investigate current topics of research. The results of neuroimaging studies have been presented as well. Attention has also been dedicated separately to specific issues, like psychiatric aspects of co-infection with $\mathrm{HCV}$ and $\mathrm{HIV}$ viruses, the chronic fatique in the course of $\mathrm{HCV}$ infection, the influence of substance use disorders and difficulties encountered during treatment with interferon. Undiagnosed psychiatric disorders, not only inevitably decrease the already rather low quality of life but also cause non-adherence with recommendations and medications regimes, contributing to a worse treatment outcome. Finally, the above disorders, when left untreated, result in higher rates of risk-taking behaviors among the infected, thus imposing a danger not only to patients themselves but also to the healthy population.
\end{abstract}

Key words: mental disorders, neuropathology, HCV infection, HIV infection

DOI

10.17219/acem/37787

\section{Copyright}

Copyright by Author(s)

This is an article distributed under the terms of the

Creative Commons Attribution Non-Commercial License

(http://creativecommons.org/licenses/by-nc-nd/4.0/) 
The increased prevalence of psychiatric disorders and cognitive impairment in the course of hepatitis $C$ virus (HCV) infection supports the concept that the inflammatory skirmish takes place on both sides of the blood/brain barrier, and that HCV causes a disease not only of the liver but also the central nervous system. ${ }^{1,2}$ Preliminary evidence for CNS involvement in $\mathrm{HCV}$ infection has been provided by the latest clinical, behavioral, biochemical and histological findings. The infected population has a significantly higher prevalence of various psychiatric disorders than the general population. ${ }^{3,4}$ Despite having a psychiatric disorder, many individuals never meet a mental health care professional. ${ }^{5}$ In a study by Batista-Neves et al. the diagnostic criteria for at least one psychiatric diagnosis had been met by as much as $49 \%$ of subjects and $37 \%$ of subjects would have had at least 2 diagnoses. Among them, $85 \%$ were unaware of having a psychiatric condition. ${ }^{5}$ It is common for anxiety disorders or depression to be a consequence of a chronic disease. In accordance with literature data, the frequency of psychiatric disorders concomitant with HCV has increased dramatically.,6 What is more, the HCV infection is significantly more prevalent in the population of psychiatric patients than in the general population. The prevalence is estimated to be 9-11 times higher, and reaches nearly $20 \%$, without discrepancies between groups, among psychiatric in- and out-patients. ${ }^{1,7}$ Drug addicts or people tending to undertake risky sexual behaviors also have a higher prevalence of psychiatric conditions and are at a higher risk of $\mathrm{HCV}$ infection as well as co-infection with HIV (human immunodeficiency virus). ${ }^{7-9}$ Co-infection is a concern of nearly one third of the HCV infected and about $70-90 \%$ in the population of IDUs (intravenous drug users). It is being considered that acquiring one of the viruses is a risk factor of being infected with the other. ${ }^{6}$ The unspecific character of the psychopathological symptoms in the HCV infection makes the process of thorough diagnostics and adequate treatment difficult. ${ }^{5}$ The clinical picture is quite often unclear, with somatic symptoms attracting all the attention; the psychiatric symptoms are non-specific and often recognized as manifestations of the viral infection. This can contribute to cognitive deficits at their onset or an episode of depression being overlooked. ${ }^{3,5}$ Untreated psychiatric disorders not only decrease the already low quality of life but also cause non-adherence, contributing to worse treatment outcomes. ${ }^{6}$ Finally, the above disorders, when left untreated, result in higher rates of risk-taking behaviors among the infected, thus imposing a danger not only to patients themselves but also to the healthy population. ${ }^{3}$ This article presents a spectrum of psychopathological symptoms and psychological dysfunctions, an outline of current theories on the neuropathology and psychiatric aspects of HCV infection treatment. The aim of this review is to shed light not only on the basic information concerning CNS pathology but also on the conclusions emerging from the studies of different authors, of various methodology, in diverse study groups and also to investigate current topics of research.

\section{Virus, prevalence, risk factors}

Hepatitis $C$ is caused by an enveloped ssRNA virus of the family Flaviviridae. There are 6 genotypes of the virus. $^{2}$ It is being estimated that $3 \%$ of the world population, that is 170 million people, is infected with HCV. 1.4\% of the Polish population is affected by hepatitis $\mathrm{C}$, but due to the asymptomatic course of the disease in most cases (75\% of recently infected patients) the percentage of infections may be higher.,10 The virus is characterized by a primitive system of enzymes, which is associated with high levels of drug resistance and difficulties in vaccine development. ${ }^{1,2}$ The heterogeneity of the virus and its high error rate of replication system explain why the immune system is unable to fully eradicate the virus and that $80 \%$ of acute hepatitis $\mathrm{C}$ patients enter the chronic phase of the infection. ${ }^{1}$ It leads in $20-30 \%$ to hepatic cirrhosis within 10 to 20 years. Hepatitis $\mathrm{C}$ also contributes to severely increased risk of hepatocellular cancer. ${ }^{1,2}$ Transfusion of blood or blood products prior to 1992, intravenous drug use and hemodialysis are the main risk factors of HCV infection. ${ }^{1}$ Groups of increased risk of infection also include non-intravenous drug users, patients with highrisk sexual practices, and patients admitted to a psychiatric hospital. ${ }^{11}$ It is being estimated that the infection is in 65-70\% transmitted through injection drug use, 15-20\% high-risk sexual behaviors and 5\% occupational exposure. ${ }^{12}$ Penitentiary institutions have an especially high prevalence of $\mathrm{HCV}$, even $17-21 \%$ of inmates are infected. ${ }^{7}$ According to the Himelhoch et al. study, $8.1 \%$ of patients with bipolar disorder and $7.1 \%$ with schizophrenia were infected vs. the $2.5 \%$ in the general population. ${ }^{13}$ A study conducted in 8 medical centers and 17 outpatient clinics in the United States of America (USA) showed that nearly $10 \%$ of schizophrenic and schizoaffective patients were infected with $\mathrm{HCV}$, compared to $5.3 \%$ controls. Moreover, this rate reached $30 \%$ among those patients who had a co-morbid substance use disorder. ${ }^{12}$ Primary care physicians and psychiatrists are on the forefront of diagnosing patients with mental disorders who are at increased risk of HCV infection.?

\section{Mental disorders comorbid to HCV}

Although the results of the studies concerning the prevalence of psychiatric disorders in the $\mathrm{HCV}$-positive population differ significantly, they are consistent in being considerably higher as compared to the general population. In a large study with $33842 \mathrm{HCV}$-positive subjects, $85 \%$ had a history of mental disorders including psychoactive substance use and $60 \%$ had a co-morbid psychi- 
atric disorder. ${ }^{3}$ Other studies indicate that up to $40 \%$ of $\mathrm{HCV}$-positive individuals meet the criteria for a current psychiatric disorder, with depression being the most common (28-50\%). ${ }^{4}$ Anxiety was reported in $18-41 \%$, bipolar disorder $9-16 \%$, psychotic disorder $17-24 \%$ and personality disorder (with antisocial the most common) in $30 \%$ of cases. ${ }^{4}$ In a study with the use of Structural Clinical Interview for DSM IV Axis I conducted among 500 infected subjects, the prevalence of psychiatric disorders was as follows: any depressive disorder - $18.2 \%$, major depressive disorder $-6.4 \%$, general anxiety disorder $-7 \%$ and panic disorder $-5.8 \%$. $^{14}$

\section{Chronic fatigue}

Chronic fatigue is the most frequently reported extra hepatic symptom. It is often accompanied by a depressive or anxiety symptomatology. ${ }^{4}$ Various studies provide data about $20 \%$ to $80 \%$ of patients affected by chronic fatigue syndrome (CFS). In the McDonald et al. study $83 \%$ of patients reported fatigue as the leading symptom. ${ }^{8}$ Compared with patients suffering from hepatitis B and alcoholic hepatitis, the prevalence of CFS is significantly higher in the HCV infected population. ${ }^{15}$ According to Swain et al. the potential causes of CFS occurrence in the course of liver diseases include hypothalamic-pituitary-adrenal axis, serotonergic transmission as well as immunological disorders. ${ }^{16}$ Neither the inflammation progress nor the cirrhosis stage correlate with CFS intensity. However, there is a significant correlation between fatigue and anxiety/depression levels. ${ }^{8,15}$ Older age, female gender, concomitant psychiatric disorders are important predictors with relevance to CFS. Low serum carnitine, creatinine and brain-derived neurotropic factor (BDNF) concentrations are considered to be biochemical markers. ${ }^{14,15}$ Studies using reliable self report measures: Beck Depression and Anxiety Inventories and the Brief Symptom Inventory indicate that $25-40 \%$ of $\mathrm{HCV}$ patients suffer from clinically relevant mental symptoms. ${ }^{4}$ Ailments such as arthralgy, fibromialgy, mialgy and chronic pain contributing to a significant decrease in physical and mental comfort as well as their quality of life, are present in even up to $50 \%$ of $\mathrm{HCV}$-positive patient's reports. ${ }^{3}$

\section{Depression, anxiety, sleep disorders}

Significant discrepancies between various studies are present in the evaluation of mood disorders prevalence. According to different authors, the depression rate varies from 10 to $60 \%$ in the HCV infected population. The HCV genotype 3a may be associated with increased risk of depression. ${ }^{3}$ Depression may not only be a symptom of the infection itself but also a reaction to the awareness of a se- vere, infectious and chronic disease. ${ }^{3}$ What is more, many of the studies are conducted among IDUs with depressive disorders often present with hepatitis $C$, thus making the etiology of mood disorders even more difficult to evaluate. ${ }^{8,10,13,15,17}$ Younger patients within 1-6 months after diagnosis present less pronounced depression. ${ }^{10}$ Anxiety disorders with the estimated prevalence of $18-38 \%$ are also more common in the HCV infected population. A correlation between the intensity of anxiety disorders and the occurrence of depression and alcoholism has been stated. ${ }^{18}$ Czarnecki et al. report an association between viremia, hepatic inflammation activity and the intensity of depression and anxiety disorders. ${ }^{19}$ Pawłowski et al. likewise stated a correlation between the level of depression, neuroticism and the viremia of $\mathrm{HCV}$ positive patients. ${ }^{10}$ Sleep disorders concern even up to $60 \% \mathrm{HCV}$-positive patients, especially with regard to sleep disruption, obstructive sleep apnea and restless leg syndrome. ${ }^{3}$

\section{Substance use and HCV infection}

A study by Mc Carthy et al. conducted among IDUs in methadone maintenance treatment reported an extremely high prevalence of $\mathrm{HCV}$ infection i.e. $96 \%$ subjects were seropositive and $62 \%$ had detectable HCV-RNA plasma levels. ${ }^{20}$ Although $\mathrm{HCV}$ seroconversion may take place anytime in the course of addiction, it usually happens after 1-3 years of drug use. ${ }^{12}$ Compared to the general population the prevalence of $\mathrm{HCV}$ infection is also high among non-IDUs. Depending on age, gender and type of used drugs it varies from 5 to $29 \% .{ }^{20}$ Although drug use complicates the course of the infection especially in the context of mental health and interferon (IFN) treatment, only alcohol has an established, synergistic with $\mathrm{HCV}$ influence on the liver disease progression. ${ }^{12}$ Studies show that a very small percentage of the infected population with comorbid psychiatric disorders does not have a concomitant substance use disorder. In a study by ElSerang et al., among trimorbid patients ( $\mathrm{HCV}$, psychiatric illness and substance use disorder), 85\% were diagnosed with a depressive disorder, $71 \%$ anxiety, $43 \%$ post-traumatic stress disorder (PTSD), 42\% psychotic and 30\% bipolar disorder. ${ }^{21}$ The psychiatric aspects of IFN therapy will be presented separately, leaving space for aspects of this treatment with regard to $\mathrm{HCV}$-positive patients with comorbid substance use disorder. There are significant limitations of IFN treatment in this population such as a potentially higher risk of psychiatric complications, non-adherence to treatment, re-infection among IDUs and a decrease in efficacy. ${ }^{20}$ There is no evidence suggesting that the substance use disorder has a significant influence on the IFN treatment itself, thus it is not necessary to condition the start of the treatment by discontinuing the patients' psychoactive substance use. ${ }^{12}$ It has been stated in a large retrospective study that among patients who did 
not receive treatment, $60 \%$ had a history of nasal or intravenous drug use and $23 \%$ were alcoholics. ${ }^{22}$ Several studies report, however, that patients with a present or past psychiatric disorder and a substance use disorder are capable of successfully completing a course of IFN therapy. ${ }^{23}$ One of those studies was carried out by Sylvestre among 50 methadone patients with HCV infection. ${ }^{24}$ Although 62\% subjects had a pre-existing psychiatric diagnosis (with depression being the most common), 30\% were clean for less than 6 months and 36\% relapsed to hard drug using before the end of the study, the end-of-treatment-response (ETR) reached 64\%. What is more, there were no significant ETR discrepancies between patients with short and long-lasting sobriety. Finally the relapse to drug use overall did not cause significant decreases of treatment outcomes. ${ }^{24}$ This suggests that the selected subjects, even with comorbid psychiatric disorders or current drug use, may be adequate candidates for IFN treatment. ${ }^{7}$ It needs to be mentioned that in Poland as well as in several other European countries, active addiction to psychoactive substances, current depression, psychosis or epilepsy are the basis for disqualification from IFN treatment. What is more, the occurrence of the above conditions during an IFN treatment course results in immediate exclusion from the program. ${ }^{25}$ IFN treatment is an indication for screening and evaluating patients for substance and alcohol use disorders. ${ }^{12}$ Alcohol Use Disorders Identification Test (AUDIT), or its abbreviated version AUDIT$\mathrm{C}$, are useful tools for identifying alcohol use disorders. The Drug Abuse Screening Test (DAST) or Two-Item Conjoint Screen (TILS) may be also carried out. ${ }^{7}$ There is a tool especially useful for primary care screening - Alcohol, Smoking and Substance Involvement Screening Test (ASSIST 2.1). It evaluates lifetime use of 10 drug 'classes', substance-specific recent use and, its severity, current global risk and injection drug risk. ${ }^{26}$

\section{Psychopharmacology in HCV infection}

Treatment of HCV infection and comorbid psychiatric disorders poses a therapeutic challenge due to the fact that most psychotropic medications (antidepressants, antipsychotics, mood stabilizers) undergo hepatic metabolism, thus imposing a risk of hepatotoxicity and a more rapid progression to cirrhosis. ${ }^{1}$ The most widely used antidepressants are SSRIs - citalopram and escitalopram are proved as safe, fluoxetine and sertraline are presumably safe. Paroxetine is associated with the risk of retinal hemorrhage. ${ }^{27}$ Various self- or clinician-rated scales such as Beck Depression Inventory (BDI) or Montgomery Asberg Depression Rating Scale can be used for the patient's screening. ${ }^{12}$ Results of BDI being equivalent to moderate and severe depression indicate that a treatment with antidepressants should be considered. ${ }^{7}$ Lithium carbonate and gabapentin are considered to be safe mood stabilizers, since they are eliminated through the kidneys. Valproic acid requires liver functions monitoring, whereas carbamazepine and oxcarbazepine may result in bone marrow suppression, thus are contraindicated during IFN therapy. ${ }^{18}$ Haloperidol, quetiapine and aripiprazole are regarded presumably safe antipsychotics. Risperidone requires liver functions monitoring, whereas olanzapine and clozapine are contraindicated. ${ }^{27}$ For managing sleep disorders, sleep-promoting agents such as antihistamines, sedative antidepressants (trazodone, mirtazapine), probenzodiazepine (zolpidem, zaleplon) are used for insomnia, modafinil for hypersomnia (may cause myelosuppression) and gabapentin for restless leg syndrome., ${ }^{3,27}$

\section{Neurocognitive dysfunctions}

Cognitive impairment associated with $\mathrm{HCV}$ infection is not progressive and does not lead to dementia. ${ }^{8,15}$ Forton et al. evaluated its prevalence on the level of $38 \% .^{8}$ They were the first to use computer-based cognitive battery among HCV infected patients. That study established the presence of selective impairments of attention, concentration and psychomotor speed in patients who had histological evidence of minimal HCV hepatitis. Those abnormalities were associated with neither fatigue and depression nor with a history of drug abuse. ${ }^{8}$ The cognitive disorders did not correlate with inflammation markers, steatosis or liver cirrhosis, HCV genotype and replication activity. Although conditions like psychoactive substance abuse, depression, hepatic encephalopathy and autoimmune disorders contribute to cognitive impairment, they do not explain its occurrence sufficiently. ${ }^{8}$ Other researchers like Weissenborn et al. also have not determined any associations between neurocognitive deficits and the above-mentioned conditions. ${ }^{28}$ What is more, studies conducted on particular populations like women, children, patients without substance abuse history have also given evidence that the neurocognitive dysfunctions are an independent occurrence. ${ }^{3}$ There have been 2 risk factors identified that exacerbate cognitive dysfunctions: HIV co-infection and IFN therapy. ${ }^{3}$ Both have been discussed separately. The deficits mainly affect attention, higher executive functions as well as concentration and memory, causing a condition called 'brain fog., Abstract thinking, mental flexibility, speed of information processing and visual-spatial processing may also be impaired. ${ }^{1,29}$ Fontana et al. reported disturbed learning, complex working memory and reaction time in 33\% of studied subjects and have confirmed the lack of association with fibrosis, drug use, lifetime psychiatric disorder or mood disturbance history. ${ }^{30}$ Decreased verbal fluency and psychomotor retardation are also characteristic in this group of patients. ${ }^{16,31}$ In standard neuropsychological tests, individuals with $\mathrm{HCV}$ exhibit deficits of mental and 
motor speed, divisibility and sustainability of attention and short-term working memory, but not intelligence quotient (IQ) or episodic memory. A similar profile of disorders may be present in the course of other liver diseases with or without cirrhosis. Nevertheless, in the course of hepatitis $C$ these changes tend to occur in significantly earlier stages. ${ }^{7}$ Computerized tests based on the latest advances in cognitive neuroscience are useful for the evaluation of cognitive functions of HIV-positive patients and seem to be equally suitable for the assessment of $\mathrm{HCV}$ infected subjects. These tests provide an opportunity to evaluate verbal working memory (with circuits including dorsolateral prefrontal cortex and striatum), processing speed, divided attention and decision-making parameters such as inclination to risk taking behaviors, impulsiveness, delayed and immediate reward. ${ }^{7}$ In discounting delayed rewards and through the executive dysfunction study, the investigators have found out that HCV-positive adults are (more than HCV-negative adults) inclined to show executive dysfunction and to discount delayed rewards, independently of history of substance abuse. HCV associated impairments in executive functions may result in dysexecutive problems in general and with pathological patterns in delay discounting and perhaps decision making, impulsiveness, risk behaviors in particular (especially in the context of spreading the infection). ${ }^{17,31}$

\section{Etiopathogenesis of CNS impairment}

The biological influence of $\mathrm{HCV}$ on CNS functioning is being emphasized though not fully explained yet. The presence of the virus has been determined in extrahepatic tissues: the lymph nodes, bone marrow, pancreas, thyroid gland, adrenals, and the CNS. ${ }^{29}$ Its genetic material has been detected in brain tissue in autopsy studies. ${ }^{8,19}$ The authors associate it with extrahepatic replication of the virus in the monocytes which are the source of microglia. ${ }^{24}$ Results from the Forton et al. study indicate dissimilarity between the virus genotypes present in blood and in brain tissue. ${ }^{32}$ Forton et al. discovered a brain specific genome variant of $\mathrm{HCV}$ with mutations in the internal ribosomal region that may be important for promoting extrahepatic replication. ${ }^{32}$ More recently, in a study of $\mathrm{HCV}$ genome derived from brain tissue, a specific mutation has been identified that was part of $10 \%$ of HCV sequences in the cerebellum and medulla. These mutations were not detectable in material from the liver or plasma of the studied patients. ${ }^{33}$ The above findings suggest that virus replication takes place also in the CNS. Fishman et al., on the other hand, determined the amount of HCV RNA in brain samples to be 1000-10000 times smaller than in samples from the liver, which was reflected in mild neuropathological signs occurring among the studied patients. ${ }^{33}$
Several mechanisms of HCV brain entry are being considered. An involvement of peripheral blood mononucleous cells, which could introduce the virus into CNS, is being proposed. ${ }^{32}$ Fletcher et al. observed that HCV in vitro infected the brain's microvascular endothelial cells (BMEC) - the main component of the blood/brain barrier - which undergo apoptosis, leading to a decrease of the endothelial barrier activity in the course of infection. That study suggests that HCV may disrupt the integrity of the blood/brain barrier in vivo as well. ${ }^{34}$ The presence and replication of $\mathrm{HCV}$ has been found also in BMEC which indicates that the blood/brain barrier may be directly involved in HCV brain pathogenesis. ${ }^{1,3}$ The secondary effects of a chronic inflammatory systemic process and the role of cytokines, which are presumably mediating the cognitive dysfunction, is an important part of the etiopathogenesis of the CNS pathology in the course of hepatitis C. ${ }^{14}$ During the chronic phase of the infection some cytokines like IFN- $\alpha$ or TNF- $\alpha$ may cross through the blood/brain barrier. It is being suggested that this could take place especially at the site of organum vasculosum laminae terminalis, influencing brain functioning. ${ }^{3}$ It is also likely that the cerebral dysfunctions are caused by the neurotoxins released by microglia activated in the course of the immune response. The neurotoxins include substances like nitric oxide and stimulating amino acids (aspartates and glutamates) which induce neuronal apoptosis and also neurosteroids (pregnenolon) that activate inhibitory brain pathways., ${ }^{2,4}$ Reports from neuroimaging are consistent with post-mortem studies and show microglial and inflammatory activation in patients with $\mathrm{HCV}^{3}$

The hepatitis $\mathrm{C}$ neurocognitive disorders character indicates both cortical and subcortical damage. ${ }^{28}$ Brain changes have been demonstrated in the basal ganglia. ${ }^{3}$ Alzheimer type 2 gliosis may be observed occasionally. It is a typical feature of a decompensated liver disease and reflects severe metabolic disturbances in the CNS. ${ }^{29}$ Another aspect of the neuropathology originated from observations made by Jones. ${ }^{3}$ He discovered that ondansetron (5-HT-3 receptor antagonist) ameliorates the comfort of patients through fatigue reduction and an increase of psychomotor speed. ${ }^{35}$ It indicates changes in serotoninergic neurotransmission of $\mathrm{HCV}$ patients with chronic fatigue. ${ }^{35}$ What is more, SPECT studies have shown decreased binding capacity of a serotonin transporter in the mesencephalon and/or dopamine transporter in the striatum in 14 out of 20 examined patients independently of their PCR status. ${ }^{34}$ Patients with disturbed monoamine transporter binding capacity achieved significantly worse results upon psychometric testing (especially with regard to mood and cognition) compared to the control group. Hence, the disturbances of monoaminergic neurotransmission may be an important factor of the CNS dysfunction in the course of $\mathrm{HCV}$ infection. ${ }^{34}$ 


\section{Neuroimaging}

Using proton magnetic resonance spectroscopy (H1 MRS) Forton et al. discovered metabolic abnormalities that manifested themselves as elevated choline/ creatine ratio in the basal nuclei and white matter in the brains of patients with chronic hepatitis C. ${ }^{32}$ The abnormalities were more pronounced in patients with cognitive impairment and were not associated with previous psychoactive substance abuse. ${ }^{32}$ Elevated choline levels are characteristic for processes with cell proliferation and changes in membrane fluidity including inflammations, particularly HIV infection and multiple sclerosis. ${ }^{34}$ H1MRS studies allowed us to exclude depression and hepatic encephalopathy as potential causes of cognitive dysfunctions because in both conditions the choline/ creatine ratio is reduced. ${ }^{8}$ Miller's study showed normal $\mathrm{N}$-acetylaspartate (NAA) concentrations in the neurons of HCV infected patients, which indicates a lack of neuronal loss. The above-mentioned compound concentration is usually decreased in a cerebral stroke or other situations with neuronal damage. ${ }^{33}$ More recent studies with the use of H1 MRS report, however, decreased levels of NAA in white matter and cortex. ${ }^{1,28}$ Thames et al. compared chronic HCV patients with a control group. They showed lower levels of NAA in bilateral parietal white matter, elevations in myoinosital in bilateral frontal white matter and also more pronounced fractional anisotropy in the striatum, greater mean diffusivity in the frontooccipital fasciculus and external capsule. Higher levels of fatigue correlated with diffusivity in the superior frontooccipital fasciculus in the HCV group. ${ }^{36}$

Furthermore, Weissenborn demonstrated EEG slowing in $\mathrm{HCV}$ infected individuals. ${ }^{28}$ Patients with chronic HCV hepatitis, as compared to healthy controls, also had lower prepulse inhibition (PPI). PPI is considered to be a psychophysiological marker of attention processing. It reflects the brain's capability to discriminate unimportant sensory stimuli. PPI enables the organism to direct attention and cognitive effort in the search for important information. ${ }^{4}$ PPI is automatic and involuntary; it is not influenced by factors such as fatigue, intellectual functioning or the level of education. A decrease in PPI indicates an attentional deficit mediated by frontal-subcortical circuity; hence, it is being considered as a useful and sensitive marker of attentional processing. ${ }^{4}$ Kramer et al. showed that $17 \%$ of the studied chronic HCV patients had delayed P300 peak latencies and reduced amplitudes, compared to appropriate age standards. P300 event-related potentials are a neurophysiological marker of cognitive processing; therefore, the above results indicate subtle but significant neurocognitive impairment. ${ }^{37}$ Interestingly, those results were independent of such factors as history of substance abuse or cirrhosis. This suggests a conclusion that the $\mathrm{HCV}$ infection acts on cerebral functioning directly. ${ }^{37}$
The cerebral blood volume (CBV) measurements relative to cerebellum (rCBV) are neuroimaging parameters that are being proposed as a non-invasive tool for assessing cerebral microcirculation impairment in HIV and HCV positive patients. ${ }^{38}$ Specific perfusion disturbances may be observed even in patients without neurological signs. ${ }^{38}$ Bladowska et al. compared cerebral perfusion alternations in HIV naive, cART treated, HIV/HCV and $\mathrm{HCV}$ positive naive patients. However, each of these groups had lower perfusion of several cortical areas, the HCV-infected (and also $\mathrm{HIV} / \mathrm{HCV}$ ) patients had the highest hypo-perfusion rates. ${ }^{38}$ These patients also showed hyper-perfusion in basal ganglia. ${ }^{39}$ The researchers suggest it to be a marker of early-stage $\mathrm{HCV}$ associated inflammation in patients with mild liver disease. ${ }^{38}$

\section{IFN and DAAs therapy}

Psychiatric symptoms and disorders are very often present on examination of patients treated with IFN. These are not only full-blown episodes of depression, mania, anxiety, paranoid psychoses or consciousness disorders but also mild, non-specific states of fatigue or impairments of concentration, attention and memory. ${ }^{40}$ The main chronic hepatitis type $C$ therapy consists of treatment with pegylated IFN alpha (peg-IFN $\alpha$ ) combined with ribavirin (RBW). It is associated with impairment of certain cognitive functions which are not related to depression or anxiety. ${ }^{41}$ Pawełczyk et al. mention the ability to memorize, recall and recognize verbal material; organize visual stimuli, efficiency and precision of processes of attention. ${ }^{40}$ The depressive disorders and deterioration of particular cognitive functions that are observed during peg-IFN $\alpha /$ RBW therapy may indicate the influence of IFN $\alpha$ on the dorsal-lateral prefrontal cortex and dorsal-frontal part of the gyrus cinguli that are related to attention processes, the parietal-occipital areas of the cortex which are associated with visual-spatial functions and the hippocampus which is involved in memory processes. ${ }^{40}$ A separate issue resulting from IFN treatment and one that is mentioned by many authors are the depressive symptoms and the sometimes severe episodes of depression. According to some studies the combination treatment with ribavirin is associated with a higher incidence of side effects, especially depression, anxiety and irritability. ${ }^{41}$ Interestingly, there are studies indicating that the occurrence of depression induced by treatment may be prognostic for better treatment outcome. Loftis et al. showed that both ETR and sustained viral response (SVR) were higher among patients who experienced depressive side effects than in patients who did not. ${ }^{40}$ Analysis of these studies raises a question: is the psychiatric history of the patient (including previous episodes of severe depression, schizoaffective disorders or substance use disorders) of relevance to the occurrence and severity of adverse 
reactions to IFN treatment? Schaefer et al. conducted a study on $81 \mathrm{HCV}$-positive patients who were treated with IFN $\alpha-2 \mathrm{a} .{ }^{42}$ Current and previous psychiatric disorders, substance use disorders and methadone substitution were taken into account. There were no significant discrepancies regarding SVR between study groups and the control population. ${ }^{42}$ What is more, no significant differences emerged while taking into account the occurrence and severity of first-time psychiatric conditions during therapy. The symptoms of depression present before or during treatment were statistically without any relation to the treatment outcome. Irritability was the most common adverse reaction. ${ }^{42}$ That study, as well as the more recent works by Scheafer et al., consistently shows that the HCV genotype is the only correlate of the SVR value. ${ }^{42}$ There are several mechanisms that are being considered as relevant to the occurrence of IFN treatment adverse reactions. It remains unclear which are most significant in the pathophysiology. The first possible mechanism concerns tryptophan (serotonin precursor) metabolism. ${ }^{41}$ The activity of indoleamine 2,3-dioxygenase, an enzyme responsible for converting tryptophan to kynurenine, increases resulting in decreased tryptophan plasma concentration. Hence the serotonin (5-HT) plasma levels become decreased, which is an important part of the genesis of depression. ${ }^{41}$ The increased concentration of kynurenine metabolites also relates to the induction of depressive disorders. It results in the excessive expression of N-Methyl-D-Aspartate receptors in the hippocampus, leading to its atrophy. ${ }^{40}$ What is more, IFN is a dopamine receptor agonist in the CNS. Levels of adrenaline and noradrenaline rise after IFN injections resulting in agitation, irritability and anxiety. During chronic administration, down-regulation of the receptors takes place, which may escalate depression. Fluctuations of dopamine levels may contribute to bradykinesia, anhedonia and a depressed mood. ${ }^{40,41}$ It is also apparent that IFN- $\alpha$ directly activates the hypothalamus-pituitary-adrenals axis (HPA) leading to an increase in secretion of $\mathrm{CRH}$ and AVP and also elevates ACTH levels. Increased levels of these hormones are characteristic of MDD (major depressive disorder) and anxiety disorders. ${ }^{16,18}$. Moreover, IFN is an activator of pro-inflammatory cytokines: IL-1, IL-6 and TNF $\alpha$. This results in the escalation of oxidative stress, neurotoxic effects and also the activation of the HPA axis. During the therapy of $\mathrm{HCV}$ and $\mathrm{HBV}$ infected patients a correlation has been observed between higher IL-6 plasma levels and the occurrence of depressive and anxiety symptoms. ${ }^{40}$ An IFN influence on depression through peptidases is also being considered. While MDD patients are known to have a decreased dipeptidyl peptidase IV (DPP IV) plasma activity, during IFN treatment DPP IV ${ }^{1}$ activity independently decreases. ${ }^{40}$ Schaefer et al. conducted another study among $36 \mathrm{HCV}$ infected subjects on IFN treatment. A part of this group was additionally treated with antidepressants. ${ }^{41}$ Some of the participants had a psychiatric history but it had no relevance to the conclusion that the use of SSRI's (citalopram in this study) is effective in the prevention and treatment of depressive IFN complications. Nevertheless, the values of SVR did not differ significantly between groups. ${ }^{41}$ On the other hand, Morasco et al. demonstrated in their study that paroxetine prophylaxis did not prevent depressive complications, however effective it was in their treatment. ${ }^{40}$ Concluding, it remains certain, (based on evidence from multiple studies) that SSRI's are effective in ameliorating depressive symptoms, thus, in many cases, enabling the continuation of IFN treatment. ${ }^{40}$

While IFN remains the core of HCV therapy, the paradigm is steadily moving from an immune-based, long-term therapy with severe side-effects and modest efficacy on to all oral, well-tolerated, short-term and more efficacious regimen with the use of direct acting antiviral agents (DAAs). ${ }^{43}$ The first DAAs were introduced in 2011 - the NS3/4A protease inhibitors (PIs): telaprevir and boceprevir. Since the major reported side effects include anemia, dysgeusia and rash, they do not add significant neuropsychiatric risk. ${ }^{43}$ Current clinical trials showed that other recently approved or approval awaiting - HCV NS5A inhibitors (daclatasvir, ledipasvir) or HCV NS5B polymerase inhibitors (sofosbuvir, deleobuvir) also lack adverse effects that could significantly deteriorate patients mental condition. ${ }^{43,44}$ However, DAAs are highly active antivirals, they have a low barrier to resistance. Thus, a combined treatment with PEG-IFN and RBV is being recommended, making the overall efficacy still dependent on individual IFN sensitivity and adverse effects tolerance. ${ }^{43}$ The breakthrough IFN-free regimens assume concurrent therapy with 2 or 3 DAAs, which makes the treatment extremely expensive, thus unavailable in most countries. While pharmaceutical generics are not yet available, a sofosbuvir 12-week regimen costs approximately $\$ 84,000 .{ }^{43}$ However, DAAs do not pose neuropsychiatric risk directly; they may interact with psychotropic agents through P450 cytochrome and p-glycoprotein. ${ }^{43,44}$ The contraindicated drugs list includes: midazolam, carbamazepine, pimozide and hypericum perforatum. Interactions between PIs and SSRIs or SNRIs may exacerbate adverse effect or decrease blood plasma concentrations. ${ }^{44}$ Thus parallel administration of telaprevir of escitalopram requires dose optimization. Boceprevir and teleprevir may also interact with psychotripic agents prone to corrected QT interval prolongation. ${ }^{44}$

\section{HCV and HIV coinfection}

Nearly one third of the HIV infected population is also infected with $\mathrm{HCV}$. The prevalence of co-infection reaches $70-90 \%$ in the group of infected through intravenous drug use. ${ }^{7} \mathrm{HIV}$ and HCV infections have a mutually negative impact on their course. HIV infection accelerates the development of liver cirrhosis. The prognosis for HIV dis- 
ease becomes significantly worse among the HCV infected subjects. ${ }^{6}$ Both viruses have effects that may contribute to CNS dysfunction. ${ }^{6,7}$ Patients in the asymptomatic HIV stage often exhibit impaired attention, speed of processing and working memory. Similar deficits were observed among pre-cirrhotic HCV patients. ${ }^{4}$ Slowed information processing, impaired attention and poor learning skills are also patterns of deficits frequently demonstrated in minor cognitive-motor disorder (MCMD) and patients with liver cirrhosis and subclinical type $C$ hepatic encephalopathy. ${ }^{4}$ The co-infection is often associated with more extensive psychoactive substance abuse. ${ }^{4}$

Reports concerning the influence of co-infection on the subject's mental state are unequivocal. Von Gisen et al. in consistence with Perry et al. did not state any decrease in cognitive performance in a group of HIV$\mathrm{HCV}$ positive patients. However, those patients showed slower reaction time and were more depressed. ${ }^{3,5}$ Ryan et al. report that the co-infection is associated with worse executive functioning, higher incidence of drug dependence and an increased risk of AIDS dementia complex. However, there were no significant differences regarding the occurrence of primary mental diseases. ${ }^{2}$ On the other hand, Clifford et al. showed in their study that patients simultaneously infected with HIV and $\mathrm{HCV}$ achieved worse results in cognitive tests and were more depressed (57 vs. $32 \%$ among HIV infected). ${ }^{6}$ Also Chermer et al. report that the HCV infection independently influences the impairment of cognitive functioning, especially learning abilities, abstract thinking and motor skills. ${ }^{6}$ Changes in the white matter were reported in the co-infected subjects, suggesting a direct relation with the presence of $\mathrm{HCV}$ in the brain. ${ }^{3}$ Letendre et al. established that the co-infection was associated with greater global cognitive impairment without relation to methamphetamine dependence. ${ }^{6}$

A study by Hilsabeck et al. confirms the significant influence of HIV-HCV co-infection on cognitive functioning. $80 \%$ of co-infected patients met the cognitive impairment criteria compared to $69 \%$ of HIV-monoinfected. Psychomotor slowing was stated in $84 \%$ of co-infected patients compared to $56 \%$ monoinfected. ${ }^{2}$ A study conducted among HIV-HCV positive methamphetamine users demonstrated more severe cognitive impairment and higher levels of immune activation markers than in the HCVnegative control group. Impaired memory was associated with higher HCV-RNA plasma levels. ${ }^{7}$ Antiretroviral HIV therapy may contribute to amelioration of cognitive functioning attributed to $\mathrm{HCV}$ infection although the association between cognitive impairment and the levels of virus in the brain requires further investigation. ${ }^{2}$ The grounds for HIV and HCV additive neuropathological effect have not been fully explained. It is being hypothesized that the HIV infection may promote extrahepatic HCV replication in macrophages. This could explain the relatively more common extrahepatic replication of $\mathrm{HCV}$ among the HCV-HIV positive subjects. ${ }^{6}$ There is some evidence suggesting that uncontrolled HIV disease may contribute to the facilitation of cell-associated brain entry of $\mathrm{HCV}$. According to Eugenin et al., there are certain blood-brain barrier abnormalities during the HIV infection that could enhance mononuclear cell brain migration. ${ }^{6}$ In accordance with the above mentioned information, the HCV infection also results in blood-brain barrier functioning impairment which could enhance CNS replication of HIV. ${ }^{2}$ The proposed term for this phenomenon is "synergistic copathogenicity". ${ }^{28}$ The plasma levels of IL-6, IL-1b and MIP1 $\beta$ cytokines are in co-infected patients associated with levels of cognitive abnormalities. This allows a hypothesis that the neuropathology of the co-infection is a result of interplay of HIV, HCV and the inflammatory host responses. ${ }^{2}$

\section{Summary}

There is evidence that psychiatric manifestations of $\mathrm{HCV}$ may become even more prevalent with the increasing life expectancy of the infected population. ${ }^{3}$ Neuroimaging and neurophysiological techniques have proved to be reliable surrogate markers for pathologies of the CNS. They are used in monitoring the progression of the disease and in the design of CNS-specific treatments in several neurodegenerative diseases such as multiple sclerosis and Alzheimer's disease. ${ }^{4}$ It would be of great advantage to adapt these techniques for the use in managing CNS involvement in HCV infection. Several neuroimaging and neurophysiological procedures have demonstrated a potential value in $\mathrm{HCV}$ infection, including the visualization of abnormalities in clinically asymptomatic patients. ${ }^{4}$ The prevalence of depression and other psychiatric disorders remains higher among HCV positive patients than in the general population. What is more, the rate of suicidal thoughts and tendencies is also significantly increased. ${ }^{3}$ This comorbidity is associated not only with lower quality of life and poorer adherence to therapeutic regimens, but also (as proved in several studies), with poorer treatment efficacy of infected patients with depression or cognitive impairment in particular. ${ }^{24,31}$ What is important, the above disorders when left untreated increase the rate of risk-taking behaviors among the infected, thus posing a threat not only to themselves but also to the healthy population. ${ }^{22,23}$ Moreover, there is evidence of increased overall mortality from any cause of the infected. In the case of patients with concomitant depression, the mortality due to liver insufficiency is also increased. Finally, the rate of successfully conducted liver transplants is significantly lower in this group. ${ }^{1}$ Considering the facts on the prevalence and influence of psychiatric disorders, including substance use disorders in HCV, it is clear that screening for them and their appropriate therapy is extremely important. 


\section{References}

1. Rifai MA, Gleason OC, Sabouni D. Psychiatric care of the patient with hepatitis C: A review of the literature. Prim Care Companion J Clin Psychiatry. 2010, 12(6).

2. Fletcher NF, McKeating JA. Hepatitis $C$ virus and the brain. J Viral Hepat. 2012;19:301-306.

3. Modabbernia A, Poustchi H, Malekzadeh R. Neuropsychiatric and psychosocial issues of patients with hepatitis $\mathrm{C}$ infection: A selective literature review. Hepat Mon. 2013;13(1).

4. Perry W, Hilsabeck RC, Hassanein TI. Cognitive dysfunction in chronic hepatitis C: A review. Dig Dis Sci. 2008;53(2):307-321.

5. Batista-Neves SC, Quarantini LC, Galvão de Almeida A, et al. High frequency of unrecognized mental disorders in HCV-infected patients. Gen Hosp Psychiatry. 2008;30:80-82.

6. Martin-Thormeyer EM, Paul RH. Drug abuse and hepatitis C Infection as comorbid features of HIV associated neurocognitive disorder: Neurocognitive and neuroimaging features. Neuropsychol Rev. 2009;19:215-231.

7. Sylvestre DL, Loftis JM, Hauser P, et al. Co-occurring hepatitis $C$, substance use, and psychiatric illness: Treatment issues and developing integrated models of care. J Urban Health. 2004;81(4):719-734.

8. Forton DM, Taylor-Robinson SD, Thomas HC. Cerebral dysfunction in chronic hepatitis C infection. J Viral Hepat. 2003;10:81-85.

9. Dróżdż W, Borkowska A, Wikłość M, et al. Chronic psychotic disorders and dementia in a patient with chronic hepatitis $C$ as a consequence of therapy with pegylated interferon-alpha and ribavirin. Case study. Farmakoter Psychiatr Neurol. 2006;2:87-92.

10. Pawłowski $T$, Małyszczak $K$, Inglot $M$, et al. Depressive symptoms in patients with chronic hepatitis C. Post Psychiatr Neurol. 2006;15(2):67-70.

11. Dinwiddie SH, Shicker L, Newman T. Prevalence of hepatitis $\mathrm{C}$ among psychiatric patients in the public sector. Am J Psychiatry. 2003;160(1):172-174.

12. Loftis JM, Matthews AM, Hauser P. Psychiatric and substance use disorders in individuals with hepatitis $C$ epidemiology and management. Drugs. 2006;66(2):155-174.

13. Himelhoch S, McCarthy JF, Ganoczy D, et al. Understanding associations between serious mental illness and hepatitis $C$ virus among veterans: A national multivariate analysis. Psychosomatics. 2009;50:30-37.

14. Navines R, Castellvi P, Moreno-Espana J, et al. Depressive and anxiety disorders in chronic hepatitis $C$ patients: Reliability and validity of the patient health questionnaire. J Affect Disord. 2012;138(3):343-351.

15. Pawłowski T, Małyszczak K, Inglot M, et al. Neuropsychiatric symptoms of chronic hepatitis C. Post Psychiatr Neurol. 2005;14(4):337-342.

16. Swain MG, Maric M. Defective corticotropin-releasing hormone mediated neuroendocrine and behavioral responses in cholestatic rats: Implications for cholestatic liver disease-related sickness behaviors. Hepatology. 1995;22:1560-1564.

17. Peixoto $B$, Lopez L, Areias J, et al. Executive functions In chronic hepatitis C virus-infected patients. Adv Clin Exp Med. 2008;17(1):53-60.

18. Lehman $\mathrm{CL}$, Cheung RC. Depression, anxiety, post-traumatic stress and alcohol-related problems among veterans with chronic hepatitis C. Am J Gastroenterol. 2002;97(10):2640-2646.

19. Czarnecki M, Inglot M, Małyszczak K, et al. Neuropsychiatric disorders among HCV infected patients - own observations. Przegl Epidemiol. 2005;59:431-438.

20. McCarthy JJ, Flynn N. Hepatitis C in methadone maintenance patients: Prevalence an public policy implications. J Addict Dis. 2001;20:19-31

21. El-Serag HB, Kunik M, Richardson $P$, et al. Psychiatric disorders among veterans with hepatitis $C$ infection. Gastroenterology. 2002; 123(2):476-482
22. Hatem C, Minello A, Bresson-Hadni S, et al. Is the management of hepatitis $C$ patients appropriate? A population-based study. Aliment Pharmacol Ther. 2005;21(8):1007-1015.

23. Sylvestre $D L$. Treating hepatitis $C$ virus infection in active substance users. Clin Infect Dis. 2005; 40:321-324.

24. Sylvestre DL. Treating hepatitis $C$ in methadone maintenance patients: An interim analysis. Drug Alcohol Depend. 2002;67:117-123.

25. Appendix no. 47 of the Law no. 59/2011/DGL President of NFZ, 10.10.2011

26. WHO ASSIST Working Group. The WHO Alcohol, Smoking and Substance Involvement Screening Test (ASSIST): Development, reliability and feasibility. Addiction. 2002;97:1183-1194.

27. Rifai MA, Indest D, Loftis J, et al. Psychiatric management of the hepatitis C patient. Curr Treat Options Gastroenterol. 2006;9(6):508-519.

28. Weissenborn K, Krause J, Bokemeyer $M$, et al. Hepatitis C virus infection affects the brain-evidence from psychometric studies and magnetic resonance spectroscopy. J Hepatol. 2004;41:845-851.

29. Murray J, Fishman SL, Ryan E, et al. Clinicopathologic correlates of hepatitis C virus in brain: A pilot study. J Neurovirol. 2008;14(1);17-27.

30. Fontana RJ, Bieliauskas LA, Back-Madruga C, et al. The HALT-C Trial Group. Cognitive function in hepatitis $C$ patients with advanced fibrosis enrolled in the HALT-C trial. J Hepatol. 2005;43:614-622.

31. Dróżdż W, Halota W, Palewicz E, et al. Cognitive functions disorders in patients with chronic hepatitis C. Neuropsychiatr Neuropsychol. 2008;3(3-4):126-132.

32. Forton DM, Karayiannis $P$, Mahmud N. Identification of unique hepatitis $C$ virus quasispecies in the central nervous system and comparative analysis of internal translational efficiency of brain, liver and serum variants. J Virol. 2004;78:5170-5183,

33. Fishman SL, Murray JM, Eng FJ, et al. Molecular and bioinformatic evidence of hepatitis $C$ virus evolution in brain. $J$ Infect Dis. 2008;197:597-607.

34. Fletcher NF, Wilson GK, Murray J, et al. Hepatitis C virus infects the endothelial cells of the blood-brain barrier. Gastroenterology, 2012;142(3):634-643.

35. Jones EA. Relief from profound fatigue associated with chronic liver disease by long-term ondansetron therapy. Lancet. 1999;354:397.

36. Thames AD, Castellon SA, Singer EJ, et al. Neuroimaging abnormalities, neurocognitive function and fatigue in patients with hepatitis $C$. Neurol Neuroimmunol Neuroinflamm. 2015;2(1):e59.

37. Kramer L, Bauer E, Funk G, et al. Subclinical impairment of brain function in chronic hepatitis C infection. J Hepatol. 2002;37:349-354.

38. Bladowska J, Knysz B, Zimny A, et al. Value of perfusion-weighted MR imaging in the assessment of early cerebral alterations in neurologically asymptomatic HIV-1-positive and HCV-positive patients. PLoS One. 2014;9(7):e102214.

39. Bladowska J, Zimny A, Knysz B, et al. Evaluation of early cerebral metabolic, perfusion and microstructural changes in HCV-positive patients: A pilot study. J Hepatol. 2013;59(4):651-657.

40. Pawełczyk T, Pawełczyk A, Białkowska J, et al. Cognitive disturbances observed in chronic hepatitis $C$ patients during pegylated interferon alpha and ribavirin therapy. Psychiatr Pol. 2008;42(6):925-941.

41. Lieb $K$, Engelbrecht MA, Gut $O$, et al. Cognitive impairment in patients with chronic hepatitis treated with interferon alpha (IFNa): Results from a prospective study. Eur Psychiatry. 2006;21:204-210.

42. Schaefer M, Hinzpeter A, Mohmand A, et al. Hepatitis C treatment in "difficult-to-treat" psychiatric patients with pegylated interferon-alpha and ribavirin: Response and psychiatric side effects. Hepatology. 2007;46(4):991-998.

43. Alexopoulou A, Karayiannis P. Interferon-based combination treatment for chronic hepatitis $C$ in the era of direct acting antivirals. Ann Gastroenterol. 2015;28(1):55-65.

44. Sockalingam $S$, Tseng A, Giguere P, et al. Psychiatric treatment considerations with direct acting antivirals in hepatitis C. BMC Gastroenterol. 2013;13:86. 